\title{
openheart Ischaemia change with revascularisation versus medical therapy in reduced ejection fraction
}

\author{
Robert J Mentz, ${ }^{1}$ Mona Fiuzat, ${ }^{2}$ Linda K Shaw, ${ }^{3}$ Afshin Farzaneh-Far, ${ }^{4}$ \\ Christopher M O'Connor, ${ }^{1}$ Salvador Borges-Neto ${ }^{5}$
}

To cite: Mentz RJ, Fiuzat M, Shaw LK, et al. Ischaemia change with revascularisation versus medical therapy in reduced ejection fraction. Open Heart 2015;2:e000284. doi:10.1136/openhrt-2015000284

- Additional material is available. To view please visit the journal (http://dx.doi.org/ 10.1136/openhrt-2015000284)

Received 21 April 2015 Revised 16 June 2015 Accepted 31 July 2015

\section{(DrossMark}

For numbered affiliations see end of article.

Correspondence to Dr Robert J Mentz; Robert.mentz@duke.edu

\section{ABSTRACT}

Objective: Nuclear imaging data demonstrate that revascularisation leads to favourable effects on ischaemia burden and improved outcomes compared with medical therapy (MT). In patients with heart failure (HF), the effects of MT versus revascularisation on ischaemia change and its independent prognostic significance requires investigation.

Methods: From the Duke Databank, we performed a retrospective analysis of 278 consecutive patients with coronary artery disease (CAD) and ejection fraction (EF) $\leq 40 \%$, who underwent 2 serial myocardial perfusion scans between 1993 and 2009. Ischaemia change was calculated for patients undergoing MT alone, or revascularisation. Cox proportional hazards regression modelling was used to identify factors associated with death/myocardial infarction (MI).

Results: The magnitude of ischeamia reduction was greater with revascularisation than with MT alone (median change of $-6 \%$ vs $0 \%, p<0.001$ ). With revascularisation, more patients experienced $\geq 5 \%$ ischaemia reduction compared with MT ( $52 \%$ vs $25 \%$, $\mathrm{p}<0.01$ ) and a similar percentage experienced $\geq 5 \%$ ischaemia worsening ( $13 \%$ vs $18 \%, p=0.37)$. After risk adjustment, $\geq 5 \%$ ischaemia worsening was associated with decreased death/MI $(\mathrm{HR}=0.58 ; 95 \% \mathrm{Cl} 0.36$ to 0.96).

Conclusions: In patients with HF with CAD, revascularisation improves long-term ischaemia burden compared with MT. Ischaemia worsening on nuclear imaging was associated with reduced risk of death/MI, potentially related to development of ischaemic viable myocardium as opposed to scar tissue.

Despite decades of research, ${ }^{1-3}$ the indications for and relative benefits of revascularisation in patients with heart failure (HF) with coronary artery disease $(\mathrm{CAD})$ remain a source of contention. ${ }^{4}$ Recent data in patients with $\mathrm{HF}$ and stable CAD have shown similar survival benefits for revascularisation compared with optimal medical therapy (MT) ${ }^{3}$ However, studies in the general population have suggested that the benefits of revascularisation are confined to those with underlying ischaemia. ${ }^{5-7} \mathrm{~A}$ nuclear substudy from COURAGE (Clinical Outcomes

\section{KEY QUESTIONS}

What is already known about this subject?

- Nuclear imaging data from the general population with coronary artery disease (CAD) demonstrate that revascularisation leads to favourable effects on ischaemia burden and improved outcomes compared with medical therapy (MT). In patients with heart failure and stable CAD, the relative effects of MT and revascularisation on ischaemia burden require further investigation, and the independent prognostic significance of ischaemia change is unclear.

What does this study add?

- In 278 consecutive patients with CAD and ejection fraction $\leq 40 \%$, who underwent two serial myocardial perfusion scans (MPS), revascularisation improved long-term ischaemia burden compared with MT. Ischaemia worsening on nuclear imaging was associated with reduced risk of death/myocardial infarction, potentially related to development of ischaemic viable myocardium as opposed to scar tissue.

How might this impact on clinical practice?

- These results highlight the importance of ischaemia worsening as a novel predictor of outcome possibly by identifying patients with underlying viability. We do not believe that our study can be used as justification for performing serial MPS and are not proposing any such fundamental change to current practice guidelines. Given the unexpected nature of these findings, a better understanding of the role of serial imaging is needed before translating these results into clinical practice.

Utilizing Revascularization and Aggressive Drug Evaluation $)^{8}$ demonstrated that $>5 \%$ left ventricular (LV) ischaemia improvement was associated with better outcomes on unadjusted analysis. A recent observational study ${ }^{9}$ found that a greater proportion of patients undergoing revascularisation had significant ischaemia reduction compared with those receiving MT. Patients in the MT 
cohort also tended to have more ischaemia worsening which independently predicted death or myocardial infarction (MI).

In patients with $\mathrm{HF}$ and stable $\mathrm{CAD}$, the relative effects of MT and revascularisation on ischaemia burden require further investigation, and the independent prognostic significance of ischaemia change is unclear. ${ }^{10}$ Since ischaemia is one of the primary drivers of decisions regarding revascularisation, clarification of these questions has significant implications for both patient management as well as healthcare utilisation. The aims of this study were twofold: (1) to compare the change in ischaemia with MT versus revascularisation as measured by serial myocardial perfusion scans (MPS) in a cohort of patients with LV systolic dysfunction (LVSD) and $\mathrm{CAD}$, and (2) to evaluate the independent prognostic utility of ischaemia change for death/MI. We hypothesised that revascularisation would result in greater reduction of ischaemia burden compared with MT and that ischaemia worsening would be associated with increased death/MI.

\section{METHODS}

\section{Study population}

We conducted a retrospective analysis of patients from the Duke Cardiovascular Disease and Nuclear Cardiology Databanks. All patients are followed at 6 months, 1 year and annually thereafter, with recording of major clinical events. ${ }^{11}$ We identified 278 consecutive patients with angiographically documented CAD and LVSD (ejection fraction (EF) $\leq 40 \%$ ), who underwent two serial MPS between 1993 and 2009 (figure 1). All patients had CAD documented by coronary angiography within 180 days of initial MPS.

\section{Stress testing and imaging}

Patients capable of exercise underwent treadmill stress testing with the Bruce protocol, unless the physician requested an alternative protocol. Patients unable to exercise underwent pharmacological stress testing. MPS was performed according to described protocols. ${ }^{12} 13$ In each patient, the same stress modality (exercise or pharmacological) was performed on the initial and follow-up MPS. Tc-99m was the radiotracer used in all studies and no patients received nitroglycerin during the MPS. The studies were independently interpreted without attenuation correction by three nuclear cardiologists blinded to treatment group.

The MPS were evaluated semiquantitatively for severity and extent of abnormalities with relative perfusion recorded in each myocardial segment $(0=$ no defect, $1=$ mild defect, $2=$ moderate defect and $3=$ severe defect) at rest and stress. The summed difference score (SDS), which is the sum of the differences between the stress and rest perfusion scores (reversible defects), was determined. At the time these data were collected, we used a 12-segment model. We used a previously reported algorithm for conversion of 12-segment perfusion scores to 17 -segment scores, which is highly correlated with expert reading of the same studies by the 17 -segment model. ${ }^{14}$ The percentage $\mathrm{LV}$ ischaemia for each scan was calculated from the SDS. ${ }^{14}$

\section{Follow-up and outcomes}

Treatment group was assigned on an intention-to-treat basis. Patients were included in the revascularisation group if percutaneous coronary intervention (PCI) or coronary artery bypass grafting (CABG) occurred within 60 days of the first scan. If no revascularisation occurred within this period, they were assigned to the MT group. Follow-up time was initiated at the second MPS (time $=0$ ). For all survival analyses, we censored data at the time of participant loss to follow-up, or the end of study event surveillance follow-up. The primary end point was a composite of death/MI. An independent, blinded clinical events committee reviewed major clinical events.

\section{Statistical analysis}

Differences in baseline characteristics were compared with the use of the $t$ test or $\chi^{2}$ statistics. When appropriate, paired $\mathrm{t}$ tests were used to compare paired data. Continuous variables that were not distributed normally were compared with a Wilcoxon rank-sum test for independent groups and a Wilcoxon signed-rank test for paired data. A threshold change in ischaemia of $\geq 5 \%$ was used, as it represents a change that exceeds test repeatability. ${ }^{15}$

Kaplan-Meier methods were used to evaluate time to death/MI. Cox proportional hazards regression modelling was used to identify factors that were associated with death/MI. After examining the results of a flexible Cox model-fitting approach involving cubic polynomial spline functions, ${ }^{16}$ the linearity of the unadjusted relationship between each continuous and ordinal variable and death/MI was assessed. The proportionality assumption was verified using Schoenfeld residuals. For the multivariable model, covariates were chosen based on known clinical risk factors as well as by stepwise selection (and backwards elimination) at $\mathrm{p}<0.05$ from the list of baseline characteristics. Candidate variables for adjustment included age, non-cardiac Charlson index, LVEF, diabetes, smoking, number of diseased vessels, New York Heart Association (NYHA) class, race, history of MI, peripheral vascular disease, cerebrovascular disease and hypertension. To explore potential modifying effects of baseline ischaemia, the interaction between $\geq 5 \%$ ischaemia improvement or worsening and ischaemia on the first scan was tested in the adjusted Cox model. An inverse probability weighting model adjustment to account for propensity for treatment was performed to investigate the association between treatment and outcome. The association between ischaemia change and outcome was also evaluated in this manner. All tests were two-tailed, and statistical significance was declared 
Figure 1 Study

population (CAD, coronary artery

disease; LVSD, left ventricular

systolic dysfunction; MPS,

myocardial perfusion scan; MT, medical therapy; Revasc, revascularisation).

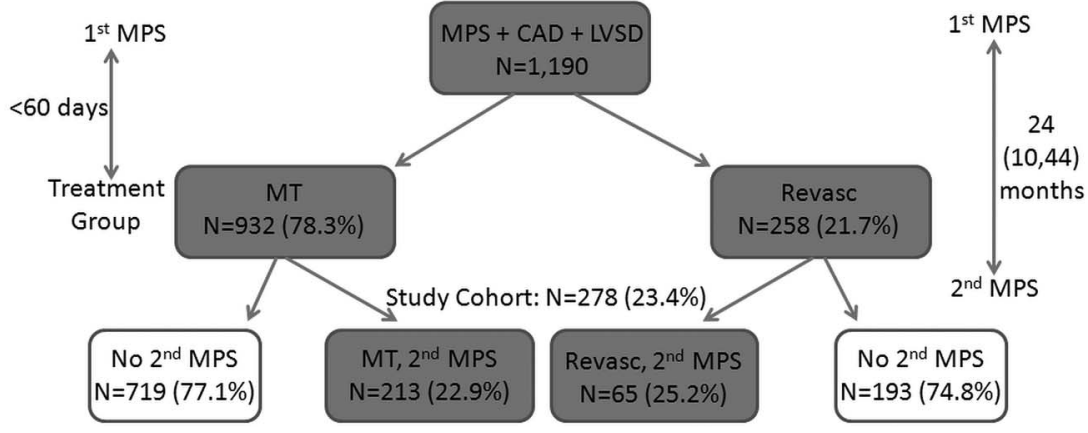

if the two-sided $\mathrm{p}$ value was $<0.05$. Most variables had very low rates of missingness (ie, <5\%). For variables with $<5 \%$ missingness, we imputed continuous variables to the overall median value, dichotomous variables to 'no', and multichotomous variables to the most frequent categorical value. For variables with $>5 \%$ missingness, we treated the missing values as a separate category. Statistical analyses were performed using SASV.9.2 (SAS Institute, Cary, North Carolina, USA). The study protocol was reviewed and approved by the Duke Institutional Review Board and all participants provided informed consent.

\section{RESULTS}

\section{Baseline clinical characteristics}

A total of 278 patients met criteria for the study with a median follow-up of 3.9 years (IQR 1.6-6.3); there was no significant difference in follow-up between the two cohorts (3.7 years (IQR 1.3-6.4) for MT vs 4.2 years (IQR 2.4-6.2) for revascularisation; $p=0.37$ ). The median time between MPS was 24 months (IQR 10-44). Baseline characteristics for the MT and revascularisation groups are provided in table 1. Of the cohort that underwent revascularisation within 60 days of the initial MPS, 47 patients underwent PCI and 18 patients

Table 1 Baseline characteristics

\begin{tabular}{|c|c|c|c|}
\hline \multirow[b]{2}{*}{ Parameter } & \multicolumn{2}{|l|}{ Treatment group } & \multirow[b]{2}{*}{ p Value } \\
\hline & Medical $(n=213)$ & Revascularisation $(n=65)$ & \\
\hline Age, years & $63(53,71)$ & $68(59,78)$ & $<0.01$ \\
\hline Male & $74 \%$ & $80 \%$ & 0.30 \\
\hline Caucasian & $72 \%$ & $71 \%$ & 0.41 \\
\hline Hypertension & $67 \%$ & $79 \%$ & 0.08 \\
\hline Hyperlipidaemia & $70 \%$ & $69 \%$ & 0.91 \\
\hline Diabetes & $30 \%$ & $48 \%$ & 0.01 \\
\hline Peripheral vascular disease & $16 \%$ & $19 \%$ & 0.57 \\
\hline Prior myocardial infarction & $70 \%$ & $51 \%$ & 0.01 \\
\hline Prior percutaneous coronary intervention & $43 \%$ & $40 \%$ & 0.70 \\
\hline Prior coronary artery bypass grafting & $50 \%$ & $39 \%$ & 0.11 \\
\hline Prior revascularisation & $69 \%$ & $59 \%$ & 0.11 \\
\hline 3-vessel disease & $54 \%$ & $57 \%$ & 0.63 \\
\hline Ejection fraction, \% & $32(26,36)$ & $33(28,37)$ & 0.05 \\
\hline Prior smoking & $67 \%$ & $63 \%$ & 0.55 \\
\hline New York Heart Association class III-IV & $25 \%$ & $20 \%$ & 0.42 \\
\hline Charlson Index & $1(0,1)$ & $1(0,2)$ & 0.06 \\
\hline 1 & $34 \%$ & $38 \%$ & 0.18 \\
\hline$\geq 2$ & $23 \%$ & $31 \%$ & \\
\hline Body mass index, $\mathrm{kg} / \mathrm{m}^{2}$ & $27(25,31)$ & $28(26,32)$ & 0.07 \\
\hline Heart rate, bpm & $73(64,85)$ & $69(61,78)$ & 0.04 \\
\hline Systolic blood pressure, $\mathrm{mm} \mathrm{Hg}$ & $132(117,149)$ & $144(127,167)$ & $<0.01$ \\
\hline$\beta$-Blocker & $52 \%$ & $51 \%$ & 0.85 \\
\hline ACE inhibitor & $62 \%$ & $66 \%$ & 0.60 \\
\hline Nitrate & $56 \%$ & $46 \%$ & 0.16 \\
\hline Statin & $25 \%$ & $44 \%$ & $<0.01$ \\
\hline Aspirin & $68 \%$ & $56 \%$ & 0.07 \\
\hline Calcium channel blocker & $16 \%$ & $28 \%$ & 0.05 \\
\hline Diuretic & $17 \%$ & $28 \%$ & 0.07 \\
\hline
\end{tabular}


underwent CABG. The main clinical indications for the second scan were chest pain $(42.1 \%)$, shortness of breath/fatigue $(25.9 \%)$ and arrhythmia $(14.0 \%)$. Other indications included syncope, preoperative testing and abnormal ECG. Of the overall nuclear study cohort with CAD and LVSD $(\mathrm{N}=1190)$, the percentage of patients with a second scan was $22.9 \%$ in the MT group and $25.2 \%$ in the revascularisation group. Put another way, for the 278 patients who had a second scan, $23.4 \%$ had undergone revascularisation $(\mathrm{N}=65)$, while for those without a second scan, $21.2 \%$ had undergone revascularisation $(\mathrm{N}=193)$. The characteristics of patients who satisfied the inclusion/exclusion criteria for the study but did not have a second scan are shown in online supplementary table S1. Of the 213 patients initially assigned to the MT group, 71 patients (33\%) subsequently underwent revascularisation during follow-up. For the 71 patients who crossed over from MT to revascularisation, $21(30 \%)$ had MI events after the initial MPS. Of these MI events, four occurred $>30$ days prior to the revascularisation cross-over, six occurred within 2 days prior to the revascularisation, two occurred on the day of revascularisation and nine occurred $>30$ days following cross-over.

\section{Pretreatment and post-treatment ischaemia}

Table 2 presents the ischaemia data at baseline and posttreatment. The total defect size as represented by the summed stress score (SSS) was greater at baseline in the revascularisation group compared with the MT group. At follow-up, the SSS tended be lower than baseline in the revascularisation group and higher than baseline in the MT group. Comparing the SSS at follow-up between the two treatment groups, there was no longer a significant difference. In both groups, there was a significant reduction in ischaemia with therapy (figure 2). However, the revascularisation group experienced a greater reduction in ischaemia compared with the MT group (figure 3). The change in ischaemia differed over the time course of the study with greater reduction in ischaemia after the year 2000 compared with before 2000 (median ischaemia reduction of $-2 \% \mathrm{LV}$ ischaemia (IQR -10 to 1 ) after the year 2000 vs $0 \% \mathrm{LV}$ ischaemia (IQR -4 to 2) before 2000, $\mathrm{p}=0.023$ ). This difference was present in the MT group $(\mathrm{p}=0.035)$, but not in the revascularisation group $(\mathrm{p}=0.85)$.

\section{Ischaemia change}

The proportion of patients with $\geq 5 \%$ ischaemia reduction was significantly greater in the revascularisation group compared with MT group (figure 4). The proportion of patients with $\geq 5 \%$ ischaemia worsening was similar in the treatment groups. The proportion of patients with $\geq 5 \%$ ischaemia worsening was not significantly different when comparing individuals in the study from before the year 2000 to those after.

Table 3 presents the summed rest score (SRS), which reflects the scar burden, in those patients with and without $\geq 5 \%$ ischaemia worsening. The SRS was similar between these two groups on the baseline scan, but the group with $\geq 5 \%$ ischaemia worsening had a lower SRS at $\operatorname{scan} 2(\mathrm{p}=0.03)$.

Table 2 Ischaemia from baseline to post-treatment based on treatment modality

\begin{tabular}{|c|c|c|c|c|}
\hline & Overall cohort & Medical therapy & Revascularisation & p Value* \\
\hline \multicolumn{5}{|l|}{ Baseline SSS } \\
\hline Mean \pm SD & $13 \pm 7$ & $13 \pm 7$ & $15 \pm 6$ & 0.015 \\
\hline Median (Q1, Q3) & $14(9,18)$ & $13(8,17)$ & $15(12,19)$ & \\
\hline \multicolumn{5}{|l|}{ Baseline SRS } \\
\hline Mean & $10 \pm 6$ & $10 \pm 6$ & $10 \pm 6$ & 0.325 \\
\hline Median & $10(5,14)$ & $10(5,15)$ & $8(5,13)$ & \\
\hline \multicolumn{5}{|c|}{ Baseline ischaemia (\%LV) calculated from SDS } \\
\hline Mean & $9 \pm 12$ & $7 \pm 11$ & $15 \pm 12$ & $<0.001$ \\
\hline Median & $5(0,14)$ & $0(0,11)$ & $13(5,22)$ & \\
\hline \multicolumn{5}{|l|}{ Post-treatment SSS } \\
\hline Mean & $14 \pm 7$ & $14 \pm 8$ & $14 \pm 7$ & 0.757 \\
\hline Median & $14(9,20)$ & $14(9,20)$ & $14(9,20)$ & \\
\hline \multicolumn{5}{|l|}{ Post-treatment SRS } \\
\hline Mean & $12 \pm 7$ & $13 \pm 7$ & $11 \pm 7$ & 0.094 \\
\hline Median & $12(7,18)$ & $12(7,18)$ & $11(6,16)$ & \\
\hline \multicolumn{5}{|c|}{ Post-treatment ischaemia (\%LV) from SDS } \\
\hline Mean & $6 \pm 10$ & $5 \pm 9$ & $8 \pm 12$ & 0.004 \\
\hline Median & $0(0,8)$ & $0(0,7)$ & $4(1,13)$ & \\
\hline \multicolumn{5}{|c|}{ Change in ischaemia (\%LV) from SDS } \\
\hline Mean & $-3 \pm 12$ & $-2 \pm 12$ & $-6 \pm 12$ & $<0.001$ \\
\hline Median & $0(-8,1)$ & $0(-6,2)$ & $-6(-12,0)$ & \\
\hline
\end{tabular}




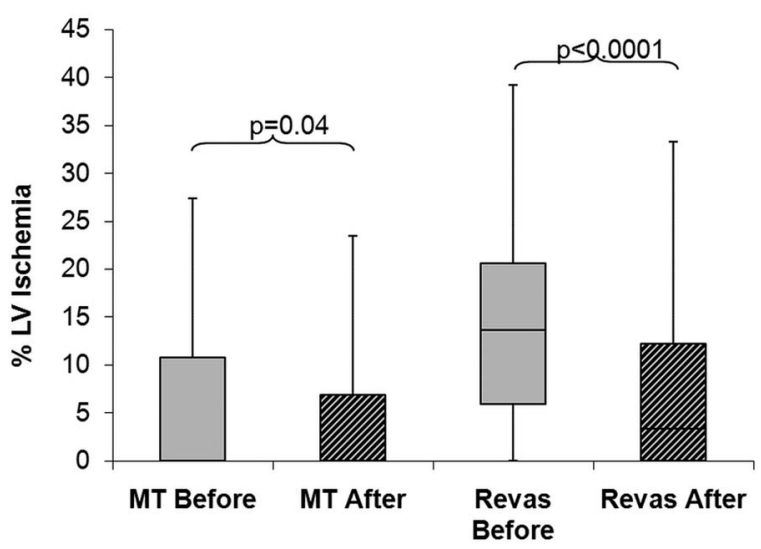

Figure 2 Percentage of LV ischaemia before and after treatment. Boxes display median, 25th and 75th centiles. Whiskers display 5th and 95th centiles. LV, left ventricular; MT, medical therapy; Revas, revascularisation.

\section{Clinical outcomes}

There was a significant difference in Kaplan-Meier event rates $(\mathrm{p}=0.03)$ between patients with $\geq 5 \%$ ischaemia worsening compared with those with $<5 \%$ ischaemia worsening (figure 5). Patients with $\geq 5 \%$ ischaemia worsening experienced lower event rates than those with $<5 \%$ ischaemia worsening. After 5 years, the composite end point of death/MI occurred in 34\% (IQR 18-50\%) of patients with $\geq 5 \%$ ischaemia worsening compared with 52\% (IQR 45-60\%) of those with $<5 \%$ ischaemia worsening. Notably, patients with $\geq 5 \%$ worsening ischaemia in the overall cohort tended to undergo revascularisation more than patients with $<5 \%$ worsening ischaemia ( $26 \%$ vs $16 \%$, respectively; $\mathrm{p}=0.12$ ). There was a non-significant trend towards lower Kaplan-Meier event rates $(p=0.13)$ in patients with $\geq 5 \%$ ischaemia reduction compared with those with $<5 \%$ ischaemia reduction.

\section{Multivariable analysis}

After adjustment for clinical and established risk predictors, as well as treatment arm, the association of ischaemia worsening $(\geq 5 \%)$ with reduced risk of death/MI remained significant. Younger age, Caucasian race and higher EF were additional predictors of decreased risk for death/MI in the main effects model. Ischaemia reduction $(\geq 5 \%)$ was not associated with the risk of death/MI $(p=0.30)$. Residual ischaemia on the final scan was not a significant independent predictor when ischaemia worsening was included in this multivariable model $(\mathrm{p}=0.27)$. Patients with $\geq 5 \%$ ischaemia worsening did not have a statistically significant difference in baseline ischaemia compared with those without $<5 \%$ ischaemia worsening (median $2.4 \%$ vs $4.9 \%, \mathrm{p}=0.51$ ). However, there was a trend towards a significant interaction between baseline ischaemia (at scan 1) and $\geq 5 \%$ ischaemia worsening in our final outcome model $(p=0.0518)$. Specifically, there was greater reduction of risk in patients with higher baseline ischaemia values (ie, the protective value of ischaemia worsening was at the higher SDS values). The HRs of ischaemia worsening at an SDS of $1,5,10$ and 15 were 0.82 (95\% CI 0.47 to $1.41), 0.63$ (95\% CI 0.38 to 1.05$), 0.46$ (95\% CI 0.24 to 0.86 ) and 0.330 (95\% CI 0.14 to 0.79 ), respectively.

The inverse probability weighting model (table 4) demonstrated that after adjustment for propensity, treatment was not significantly associated with outcome $(\mathrm{p}=0.13)$. Also, in this setting, the association between worsening ischaemia and outcomes was unchanged $(p=0.03)$. The only difference between the propensity adjusted model and the unadjusted model was that EF was no longer significantly associated with outcome following propensity adjustment. Given that the size of the baseline defect could represent a confounding factor in the ability to detect a change over time, we performed an additional analysis adding baseline SSS and SDS into
Figure 3 Median ischaemia change with treatment. Boxes display median, 25th and 75th centiles. Whiskers display 5 th and 95th centiles. LV, left ventricular; Revasc, revascularisation.

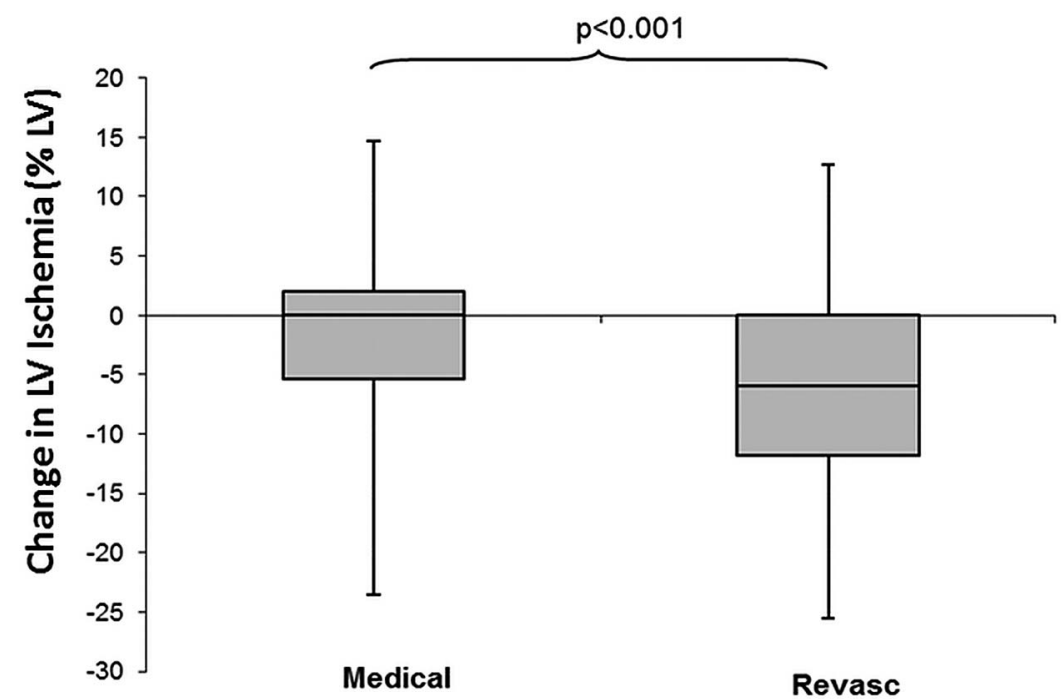




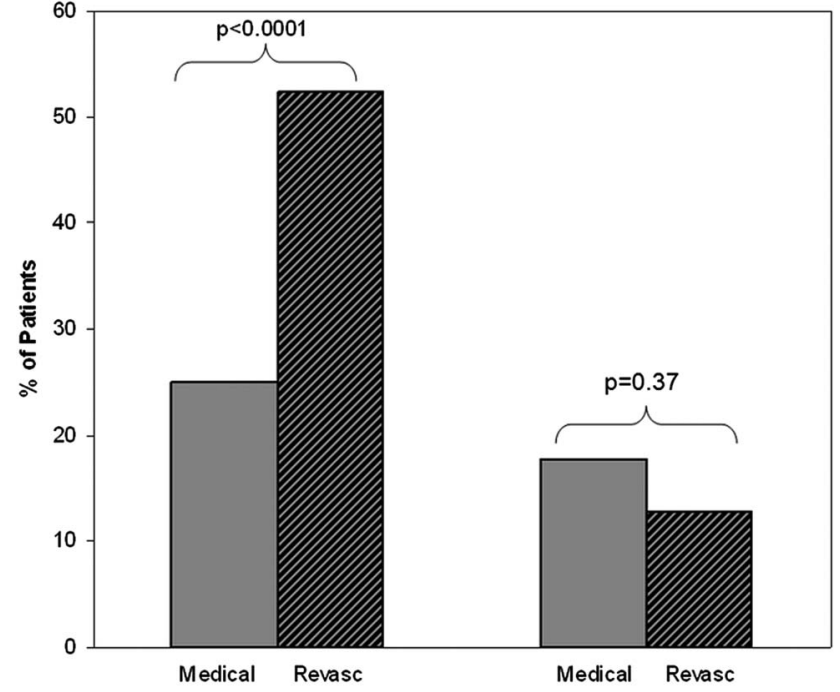

Ischaemia Reduction Ischaemia Worsening

Figure 4 Percentage of patients with $\geq 5 \%$ reduction or worsening in LV ischaemia by treatment group (Revasc, revascularisation).

the multivariable model. The model was essentially unchanged and neither baseline SSS or SDS were significant in this setting (data not shown).

\section{DISCUSSION}

This observational study assessed changes in ischaemia on serial MPS in patients with LVSD and CAD. Treatment with MT or revascularisation both resulted in overall ischaemia reduction; however, the magnitude of ischaemia reduction was greater with revascularisation. With revascularisation, more than twice as many patients experienced $\geq 5 \%$ ischaemia reduction and a similar percentage experienced $\geq 5 \%$ ischaemia worsening compared with MT. Ischaemia reduction was not associated with the risk of death/MI. In contrast, ischaemia worsening was significantly associated with a $42 \%$ reduced risk of death/MI. After adjustment for propensity for treatment, the association between ischaemia worsening and outcome persisted. Treatment strategy was not significantly associated with outcome.

The association between ischaemia worsening and improved outcomes was unexpected, particularly when considering the previous COURAGE results suggesting better outcomes on unadjusted analysis in those with ischaemia reduction. ${ }^{8}$ There are several potential

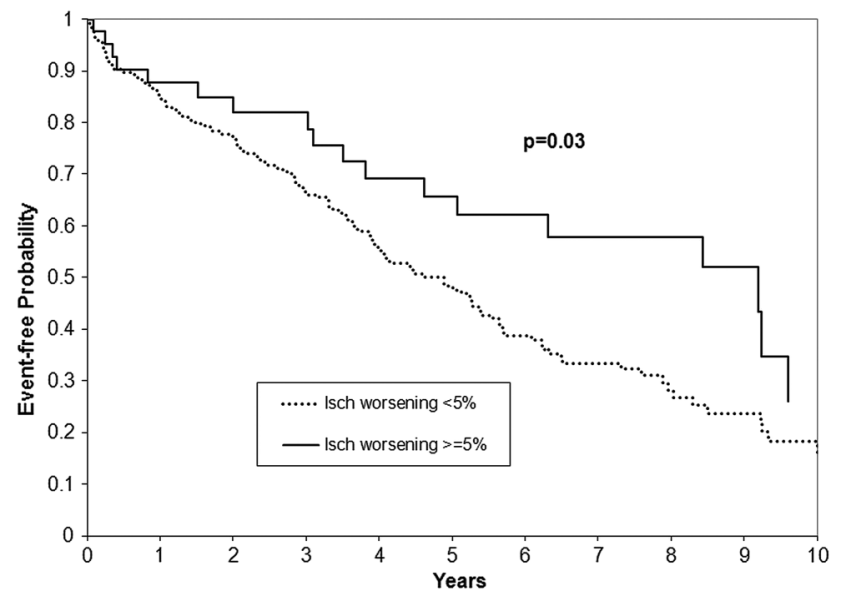

Figure 5 Kaplan-Meier event-free curve for death/ myocardial infarction for patients with $\geq 5 \%$ worsening ischaemia (isch) compared with those with $<5 \%$ ischaemia worsening.

explanations for the association of reduced risk in patients with LVSD with worsening ischaemia. First, patients that develop worsening ischaemia may be more likely to have viable myocardium as opposed to myocardial scar. Our observation of a lower SRS (ie, lesser extent and severity of perfusion defect at rest) in those patients with $\geq 5 \%$ ischaemia worsening supports this hypothesis. The presence of viable myocardium was associated with a greater likelihood of survival in patients with CAD and LVSD in the STICH trial (Surgical Treatment for Ischemic Heart Failure). ${ }^{3}$ However, this relationship was not significant after adjustment for other baseline variables. Another possibility is that in a subset of patients with LVSD and CAD, underlying ischaemia may actually reduce events due to ischaemic preconditioning'. ${ }^{17} 18$ Ischaemic preconditioning refers to the ability of short periods of ischaemia and reperfusion to increase the resistance of the myocardium to a subsequent ischaemic insult. Third, those patients with worsening ischaemia and more prominent symptoms may be more likely to receive closer physician follow-up with increased attention to secondary prevention measures.

\section{Comparison with COURAGE substudy}

Similar to our findings, the COURAGE substudy ${ }^{8}$ showed that mean ischaemia reduction with PCI was greater than with MT alone and that a greater proportion of patients undergoing PCI had significant

\begin{tabular}{lcc}
\multicolumn{2}{c}{ Table 3} & Summed rest score (SRS) values in patients with and without $\geq 5 \%$ ischaemia worsening \\
\hline & $<5 \%$ Ischaemia worsening & $\geq 5 \%$ Ischaemia worsening \\
\hline $\begin{array}{l}\text { Scan 1 } \\
\text { SRS; median (Q1, Q3) }\end{array}$ & $14(7,20)$ & $11(5,18)$ \\
Scan 2 & $12(7,19)$ & $9(7,14)$ \\
$\quad$ SRS; median (Q1, Q3) & & 0.25 \\
\hline
\end{tabular}


Table 4 Adjusted relationship of treatment and ischaemia change with outcome (death and myocardial infarction)

\begin{tabular}{lllll}
\hline Predictors & $\chi^{2}$ & HR & 95\% Cl & p Value \\
\hline Age at second scan (per 10 year increase) & 10.16 & 1.28 & 1.10 to 1.49 & 0.44 to 0.97 \\
Caucasian & 4.52 & 0.65 & 0.01 & 0.03 \\
$\geq 5 \%$ worsening ischaemia & 4.46 & 0.58 & 0.96 & 0.03 \\
Ejection fraction (per 5\% increase) & 2.45 & 0.91 & 0.12 \\
Revascularisation within 60 days & 2.31 & 0.76 & 0.53 to 1.08 & 0.13 \\
Number of diseased vessels & 0.04 & 1.02 & 0.83 to 1.33 \\
\hline
\end{tabular}

ischaemia reduction. Furthermore, $\geq 5 \%$ ischaemia reduction was not associated with a decreased risk of death/MI on adjusted analysis in both studies. The proportion of MT patients achieving $\geq 5 \%$ ischaemia reduction in COURAGE $(19 \%)$ was similar to our study $(25 \%)$ despite the fact that ours was an observational experience. In contrast, over $50 \%$ of the revascularisation patients in our study had a $\geq 5 \%$ reduction in ischaemia compared with $33 \%$ in the COURAGE trial. This difference may relate to the greater baseline ischaemia in our revascularisation group (mean of $15 \%$ ) compared with that in the COURAGE substudy (mean of $8.2 \%$ ) and to the fact that our revascularisation group also included those receiving CABG. The overall crossover rate from MT to revascularisation was 33\% in our study, which was similar to that in the COURAGE and the Bypass Angioplasty Revascularization Investigation 2 Diabetes (BARI-2D) trials. ${ }^{19} 20$

It should be noted that there are important differences between the present study and the COURAGE nuclear substudy. First, our study involves those with LVSD which were excluded from COURAGE; patients with NYHA III-IV symptoms were also excluded from BARI-2D. Moreover, this is an observational study of prospectively collected data and not a randomised trial. Therefore, there were baseline differences between the treatment groups. Second, the MPS in our study were ordered at the discretion of the individual physicians caring for these patients and not routinely as prescribed in a clinical trial. Third, patients in COURAGE were taken off their antianginal medications for the baseline scan. In our study, there was no specific change in medications during their scans. Lastly, in contrast to COURAGE, our study population included the full range of stable patients with $\mathrm{CAD}$, including those undergoing $\mathrm{CABG}$ and those with prior revascularisation procedures.

\section{Comparison with STICH substudy}

These results should also be contrasted with a recent ischaemia substudy of the STICH trial. ${ }^{21}$ This analysis investigated a subset of 399 patients (33\% of the cohort) who underwent either a MPS or a dobutamine stress echo within 90 days of randomisation. The significance of baseline stress-induced ischaemia in patients with LVSD and CAD was assessed with respect to clinical outcomes and the ability to identify those patients most likely to benefit from revascularisation. There was no difference in clinical outcomes between those patients with and without ischaemia. There was also no interaction between the presence of ischaemia and the treatment effect of CABG for any of the clinical end points. Our results are distinct from these findings since they demonstrate the prognostic significance of ischaemia change over time and included the patient population undergoing PCI. Second, the number of patients with moderate-to-severe ischaemia in the STICH analysis was quite small in comparison to our cohort. In addition, while there are limitations with respect to the patient selection process in our analysis, a potentially different set of limitations has been discussed for the STICH analysis. For instance, the baseline characteristics of the ischaemia substudy population demonstrated more advanced disease (eg, lower EF and larger LV volumes) than the overall STICH cohort. These findings suggest bias in the selection of patients for the performance of stress testing in the context of a clinical trial, which may be different than in routine clinical use.

Several other studies have assessed ischaemia change or residual ischaemia during treatment of $\mathrm{CAD},{ }^{22-24}$ but none examined their independent prognostic significance or impact in the HF population.

\section{Clinical implications}

In current clinical practice, risk assessment of patients with LVSD and stable CAD is based on a combination of clinical factors, number of diseased vessels and amount of ischaemia on objective stress testing. However, it has previously been shown that the amount of ischaemia is actually a relatively weak predictor of death and a much better predictor of ischaemic events such as non-fatal MI, unstable angina and revascularisation. ${ }^{25}{ }^{26}$ Nevertheless, amount of ischaemia is one of the primary measures driving decisions regarding revascularisation.

We have shown that the absolute amount of residual ischaemia was not a significant predictor of events when adjusted to the effects of ischaemia worsening and other clinical factors. This is not surprising since the majority of events in our population were deaths (after 1 year29 deaths and 10 MIs; after 2 years-45 deaths and 13 MIs) and ischaemia largely predicts MIs not death. In contrast, worsening ischaemia was significantly associated with decreased events regardless of treatment and other clinical factors. These results highlight the importance 
of ischaemia worsening as a novel predictor of outcome possibly by identifying patients with underlying viability.

We do not believe that our study can be used as justification for performing serial MPS and are not proposing any such fundamental change to current practice guidelines. Given the unexpected nature of these findings, a better understanding of the role of serial imaging is needed before translating these results into clinical practice. In the future, incorporation of modalities like MR stress perfusion into these studies would be of particular interest to address concerns regarding repeated radiation exposure. However, the use of implantable devices in patients with low EF represents one potential limitation.

\section{Limitations}

Our study should be interpreted in the context of several limitations. First, this was a retrospective analysis. There were significant differences between those who received MT versus revascularisation with respect to diabetes and prior MI. While these factors were included in the candidate variable list for adjustment, other measured and unmeasured factors may have influenced these results. Given the modest sample size of the present analysis, and the potential residual confounding including patient cross-over with subsequent revascularisation, these data need to be replicated in larger prospective studies. Future studies will also need to explore whether these findings are also observed in those with more severe baseline ischaemia. Data were unavailable regarding $\mathrm{LV}$ volume which warrants future investigation in the context of the recent STICH analysis. Since the majority of events in this analysis were death rather than MIs, there is also the possibility of ascertainment bias. Our study encompassed a span of about 16 years during which there have been changes in MT and treatment goals as well as revascularisation technologies. The MT group was defined by the lack of revascularisation within 60 days of the initial scan with medication use based on provider discretion. Evidence-based medication use including $\beta$-blockers, ACE inhibitors and statin therapy was modest in this cohort. There was also likely selection bias, since all patients were seen at a single academic medical centre and all underwent cardiac catheterisation. However, to some extent, the patients in this study are more representative of the population seen in daily clinical practice. They include those with the full range of CAD severity, as well as prior revascularisations including CABG, which are often excluded from clinical trials. We have therefore provided a description of the changes in ischaemia with treatment of CAD and HF that are seen in everyday clinical practice.

\section{CONCLUSION}

In this observational study of patients with LVSD and stable CAD undergoing serial MPS, the magnitude of ischaemia reduction and the percentage of patients with $\geq 5 \%$ ischaemia reduction were significantly greater with revascularisation compared with MT. We have shown that $\geq 5 \%$ ischaemia worsening was significantly associated with reduced death and MI, potentially reflecting development of ischaemic viable myocardium rather than 'scar' tissue. Treatment strategy was not significantly associated with outcome. Further investigation is needed to prospectively evaluate these findings in order to better inform clinicians regarding treatment strategy.

Author affiliations

${ }^{1}$ Division of Cardiology, Duke University Medical Center (DUMC), Durham, North Carolina, USA

${ }^{2}$ Division of Clinical Pharmacology, DUMC, Durham, North Carolina, USA

${ }^{3}$ Duke Clinical Research Institute, Durham, North Carolina, USA

${ }^{4}$ Section of Cardiology, University of Illinois at Chicago, Chicago, Illinois, USA

${ }^{5}$ Department of Radiology, DUMC, Durham, North Carolina, USA

Contributors SB-N was involved in conception and design of the research and acquisition of the data. LKS, SB-N and RJM were involved in analysis and interpretation of the data. LKS was involved in statistical analysis. RJM was involved in drafting of the manuscript. MF, SB-N, LKS, AF-F, CMO and SB-N were involved in critical revision of the manuscript for important intellectual content. CMO and SB-N were involved in supervision.

Funding RJM is supported by the National Institute of General Medical Sciences (grant number T32GM086330)

Competing interests MF, CMO and SB-N received funding from GE Healthcare and Astellas. CMO is a consultant for GE Healthcare. SB-N is in speakers' boards for GE Healthcare and Astellas as well as advisory boards for Astellas.

\section{Patient consent Obtained.}

Ethics approval Duke IRB.

Provenance and peer review Not commissioned; externally peer reviewed.

Data sharing statement Additional unpublished data referred to in the text are available to reviewers and readers at request.

Open Access This is an Open Access article distributed in accordance with the Creative Commons Attribution Non Commercial (CC BY-NC 4.0) license, which permits others to distribute, remix, adapt, build upon this work noncommercially, and license their derivative works on different terms, provided the original work is properly cited and the use is non-commercial. See: http:// creativecommons.org/licenses/by-nc/4.0/

\section{REFERENCES}

1. O'Connor CM, Velazquez EJ, Gardner LH, et al. Comparison of coronary artery bypass grafting versus medical therapy on long-term outcome in patients with ischemic cardiomyopathy (a 25-year experience from the Duke cardiovascular disease databank). Am J Cardiol 2002;90:101-7.

2. Cleland JG, Calvert M, Freemantle N, et al. The heart failure revascularisation trial (HEART). Eur J Heart Fail 2011;13: 227-33.

3. Velazquez EJ, Lee KL, Deja MA, et al. Coronary-artery bypass surgery in patients with left ventricular dysfunction. $N$ Engl $J$ Med 2011;364:1607-16.

4. Ghali JK. CABG in patients with left ventricular dysfunction. $N$ Engl $J$ Med 2011;365:468; author reply 470-461.

5. Hachamovitch R, Hayes SW, Friedman JD, et al. Comparison of the short-term survival benefit associated with revascularization compared with medical therapy in patients with no prior coronary artery disease undergoing stress myocardial perfusion single photon emission computed tomography. Circulation 2003;107:2900-7.

6. Hachamovitch R, Rozanski A, Hayes SW, et al. Predicting therapeutic benefit from myocardial revascularization procedures: are measurements of both resting left ventricular ejection fraction and stress-induced myocardial ischemia necessary? J Nucl Cardiol 2006;13:768-78. 
7. Hachamovitch R, Rozanski A, Shaw LJ, et al. Impact of ischaemia and scar on the therapeutic benefit derived from myocardial revascularization vs. Medical therapy among patients undergoing stress-rest myocardial perfusion scintigraphy. Eur Heart $J$ 2011;32:1012-24.

8. Shaw LJ, Berman DS, Maron DJ, et al. Optimal medical therapy with or without percutaneous coronary intervention to reduce ischemic burden: results from the clinical outcomes utilizing revascularization and aggressive drug evaluation (COURAGE) trial nuclear substudy. Circulation 2008;117:1283-91.

9. Farzaneh-Far A, Phillips HR, Shaw LK, et al. Ischemia change in stable coronary artery disease is an independent predictor of death and myocardial infarction. JACC Cardiovasc Imaging 2012;5:715-24.

10. Farzaneh-Far A, Borges-Neto S. Ischemic burden, treatment allocation, and outcomes in stable coronary artery disease. Circ Cardiovasc Imaging 2011;4:746-53.

11. Harris PJ, Lee KL, Harrell FE Jr, et al. Outcome in medically treated coronary artery disease. Ischemic events: nonfatal infarction and death. Circulation 1980;62:718-26.

12. Borges-Neto S, Pagnanelli RA, Shaw LK, et al. Clinical results of a novel wide beam reconstruction method for shortening scan time of tc-99m cardiac spect perfusion studies. J Nucl Cardiol 2007; 14:555-65.

13. Borges-Neto S, Tuttle RH, Shaw LK, et al. Outcome prediction in patients at high risk for coronary artery disease: comparison between 99mtc tetrofosmin and 99mtc sestamibi. Radiology 2004;232:58-65.

14. Salerno M, Elliot L, Shaw LK, et al. Prognostic validation of an algorithm to convert myocardial perfusion spect imaging data from a 12-segment model to a 17-segment model. J Nucl Cardiol 2009;16:605-13.

15. Shaw LJ, Narula J. Risk assessment and predictive value of coronary artery disease testing. J Nucl Med 2009:50:1296-306.

16. Stone CJ, Koo CY. Additive splines in statistics. In Proceedings of the Statistical Computing Section ASA. Washington DC:1985:45-8.
17. Kloner RA, Shook T, Przyklenk K, et al. Previous angina alters in-hospital outcome in TIMI 4. A clinical correlate to preconditioning? Circulation 1995;91:37-45.

18. Abete P, Ferrara N, Cacciatore F, et al. Angina-induced protection against myocardial infarction in adult and elderly patients: a loss of preconditioning mechanism in the aging heart? $\mathrm{J} \mathrm{Am} \mathrm{Coll} \mathrm{Cardiol}$ 1997;30:947-54.

19. Boden WE, O'Rourke RA, Teo KK, et al. Optimal medical therapy with or without pci for stable coronary disease. N Engl J Med 2007;356:1503-16.

20. Frye RL, August $\mathrm{P}$, Brooks MM, et al. A randomized trial of therapies for type 2 diabetes and coronary artery disease. $N$ Engl J Med 2009;360:2503-15.

21. Panza JA, Holly TA, Asch FM, et al. Inducible myocardial ischemia and outcomes in patients with coronary artery disease and left ventricular dysfunction. J Am Coll Cardiol 2013;61:1860-70.

22. Berman DS, Kang X, Schisterman EF, et al. Serial changes on quantitative myocardial perfusion spect in patients undergoing revascularization or conservative therapy. J Nucl Cardiol 2001;8:428-37.

23. Parisi AF, Hartigan PM, Folland ED. Evaluation of exercise thallium scintigraphy versus exercise electrocardiography in predicting survival outcomes and morbid cardiac events in patients with single- and double-vessel disease. Findings from the angioplasty compared to medicine (ACME) study. J Am Coll Cardiol 1997;30: 1256-63.

24. Dakik HA, Kleiman NS, Farmer JA, et al. Intensive medical therapy versus coronary angioplasty for suppression of myocardial ischemia in survivors of acute myocardial infarction: a prospective, randomized pilot study. Circulation 1998;98:2017-23.

25. Mudrick DW, Velazquez E, Borges-Neto S. Does myocardial perfusion imaging provide incremental prognostic information to left ventricular ejection fraction? Curr Cardiol Rep 2010;12:155-61.

26. Hachamovitch R. Assessing the prognostic value of cardiovascular imaging: a statistical exercise or a guide to clinical value and application? Circulation 2009;120:1342-4. 